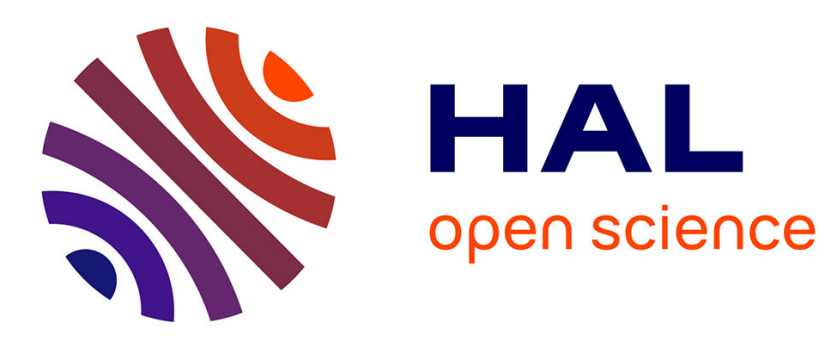

\title{
LASER INTERACTION : THERMAL AND MECHANICAL COUPLING TO TARGETS
}

\author{
R. Root
}

\section{To cite this version:}

R. Root. LASER INTERACTION : THERMAL AND MECHANICAL COUPLING TO TARGETS. Journal de Physique Colloques, 1980, 41 (C9), pp.C9-59-C9-73. 10.1051/jphyscol:1980909 . jpa00220563

\section{HAL Id: jpa-00220563 https://hal.science/jpa-00220563}

Submitted on 1 Jan 1980

HAL is a multi-disciplinary open access archive for the deposit and dissemination of scientific research documents, whether they are published or not. The documents may come from teaching and research institutions in France or abroad, or from public or private research centers.
L'archive ouverte pluridisciplinaire HAL, est destinée au dépôt et à la diffusion de documents scientifiques de niveau recherche, publiés ou non, émanant des établissements d'enseignement et de recherche français ou étrangers, des laboratoires publics ou privés. 


\title{
LASER INTERACTION : THERMAL AND MECHANICAL COUPLING TO TARGETS
}

\author{
R.G. Root
}

Physical Sciences Ine. Wobum, MA 01801 U.S.A.

Abstract.- The physical phenomena which influences thermal and mechanical coupling of infrared laser radiation to materials are reviewed. Both pulsed and $\mathrm{CW}$ interactions are considered, but the interaction of pulsed lasers with metals in an air environment is emphasized. Selected examples of vacuum interactions and coupling to non-metals are also included.

\section{INTRODUCTION}

When a pulsed laser beam irradiates a surface, the fraction of the incident energy coupled locally into the target and the impulse imparted to the target vary strongly according to which physical phenomena dominate the interactions with the target. For example, at low laser intensities the thermal energy deposited in a metal surface is controlled by the intrinsic absorptivity of the metal. However, at higher intensities, where an air plasma is ignited, the fraction of the laser energy transferred to the surface locally can be dramatically increased. ${ }^{1,2}$ This effect is called the enhanced thermal coupling. At even higher intensities, the local coupling decreases and may fall below the intrinsic absorptance. ${ }^{1,2}$ Thus, the fraction of energy deposited in a material is a sensitive function of the laser parameters - intensity, pulse time, and spot size. The purpose of this paper is to review the physical phenomena which influence the thermal and mechanical coupling of laser radiation to materials.

The approach which is followed is: (1) to choose a few important interactions, (2) to describe them briefly, and (3) to illustrate the important effect with selected experimental results. Because of the number of laser wavelengthes, target materials, ambient conditions and laser pulse intensities is overwhelmingly large, the scope of this review is limited to infrared lasers $(10.6 \mu \mathrm{m}$ and $3.8 \mu \mathrm{m})$ and to intensities below $10^{8} \mathrm{w} / \mathrm{cm}^{2}$. Metals are the primary materials considered, but selected non-metals are included in some interaction regimes. The interactions generally occur in air at standard conditions, except for a few examples of vacuum interactions. Analysis of the material response is limited to the changes which occur during the laser pulse time which affect the laser/material surface interaction. The separation of $\mathrm{CW}$ interactions from pulsed interactions is based on the following arbitrary criterion: a $\mathrm{CW}$ interaction is insensitive to temporal variations in laser intensity; conversely, a pulsed laser interaction depenas not only on average intensity but also on temporal variations.

2. CW INTERACTIONS: THERMAI COUPLING

At low laser intensity, laser radiation interacts with a material by direct absorption; the fraction of energy coupled to the surface (hereafter called the thermal coupling coefficient) is given by the intrinsic absorptivity of the material. In this coupling regime, the thermal coupling coefficient depends only on the laser wavelength and the 
target material; laser parameters such as intensity, fluence and spot size are irrelevant. Thus, the coupling coefficient can be determined with any set of laser parameters, provided they fall within the intrinsic coupling regime.

However, as the laser intensity is increased, new phenomena occur, such as target heating, 'target mass removal, vaporization and plasma formation, which modify the coupling and introduce a dependence on laser parameters. If the intrinsic absorptivity is a function of the surface temperature, the instantaneous local absorbed energy flux is still represented by the product of the intrinsic absorptivity of the surface at the local temperature and the instantaneous local incident intensity, but the local temperature depends upon the history of absorbed energy flux over the entire laser beam interaction axea. Thus, the thermal coupling coefficient in general depends on all the laser parameters, and expeximental data can be understood only by solving the coupled problem of the target thermal response to the absorbed energy flux and the change of absorbed energy flux with target temperature. This effect occurs, for example, in aluminum. klosterman ${ }^{3}$ observed an effective coupling coefficient of .079 for vaporizing thin aluminum foils with $10.6 \mu \mathrm{m}$ laser radiation, whereas the intrinsic room temperature absorptivity is only .03 . Theoretical calculations ${ }^{4}$ predict an effective coupling of . 11 which is in reasonable agreement with the data.

If the target reaches high enough temperature to induce mass loss, the energy carried away by the removed material must be properly accounted for in order to determine the absorbed energy from experimental data or to predict theoretically the energy remaining in the material. Mass can be removed by several processes; for example, vaporiza- tion, melt removal and pyrolysis. Even when the mass removal mechanisms do not affect the local instantaneous coupling, they may still affect data interpretations. Experimental measurements of the fluence required to vaporize thin titanium foils showed a sharp increase in fluence as the laser intensity increased; this was interpreted as a transition from a regime in which melt removal dominated the mass removal process but was accompanied by vaporization of melted droplets to a regime in which complete vaporization occurred. ${ }^{3}$

The most dramatic alteration of the thermal coupling occurs when a plasma is created over a surface. The dynamics of a laser-produced plasma above a surface will depend upon the intensity of the incident laser pulse and the pulse duration. At laser intensities slightly greater than the plasma threshold intensity, a laser-supported combustion (LSC) wave $^{5}$ is usually ignited. LSC waves are often seen at intensities from $2 \times 10^{4} \mathrm{~W} / \mathrm{cm}^{2}$ $10^{6} \mathrm{~W} / \mathrm{cm}^{2}$ for $10.6 \mathrm{\mu m}$ radiation with both pulsed and $\mathrm{CW}$ laser beams. The ignition of an LSC wave initially takes place in the target vapor. 6,7 The heated target vapor subsequently transfers its energy to the surrounding air. Once the air begins to absorb a significant fraction of the laser energY, the LSC wave propagates into the air along the beam path.

The nature of the coupling in the plasma regime depends on intensity, spot size, pulse time, ambient air pressure and the target material. For long pulse times and low intensity the creation of a LSC wave at $10.6 \mathrm{~mm}$ usually results in curtailing the thermal coupling as the ISC wave propagates toward the laser flux. Thermal coupling for shot pulse times and high incident intensity is treated as a pulsed interaction.

It remains to determine the time at which va- 
por plasma ignition occurs. The observed ignition time is the sum of the time required to produce the vapor and the time required to breakdown the vapor. The vapor production time is determined from the target thermal response to the direct absorption of laser radiation. The vapor breakdown time is determined from the heating of the vapor by inverse Bremsstrahlung absorption of laser radiation. The dependence of the breakdown time on laser intensity and spot size is illustrated in Fig. 1 which compares the theoretical predictions of Pirri ${ }^{6}$ to the experimental data of Klosterman. ${ }^{3}$ Theoretical predictions of breakdown times are shown for 1-D planar vapor dynamics, and two-dimensional (axisymmetric) vapor dynamics for a laser spot radius of $.5 \mathrm{~cm}$. In the experiments the laser intensity was changed by changing the spot size. Thus, at low intensity, where the spot, radius in $1 \mathrm{~cm}$, the data agrees with the one-dimensional prediction, whereas at higher intensities, as the spot size shrinks to $0.25 \mathrm{~cm}$ and the vapor dynamics becomes two-dimensional, the data tends towards the $2-D$ prediction.

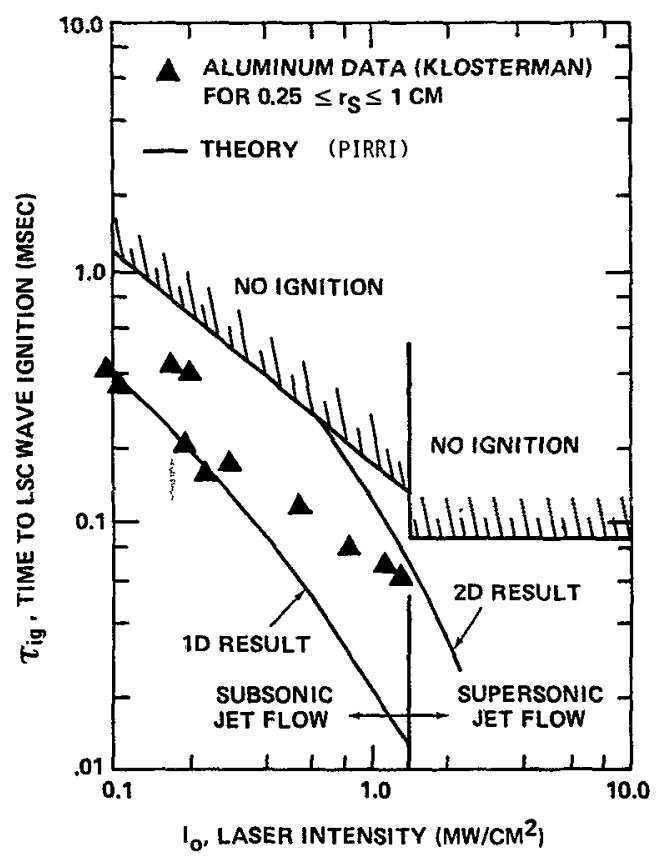

Fig. I Time to ignite laser-supported combustion wave vs. laser intensity, from Ref. 6.
Experiments at $5.0 \mu \mathrm{m}$ indicate that the vapor breakdown time scales as (wavelength) ${ }^{-2}$, as expected from inverse Bremsstrahlung absorption. ${ }^{3,6}$

3. CW INTERACTIONS - MECHANICAL COUPLING

Bulk vaporization of target material generates surface pressure and impulse. The vapor pressure on the surface depends on the ambient pressure, the laser intensity $I(t)$, laser spot radius and the detailed thermal response on the target. At high intensity, where the background pressure is irrelevant, or in vacuum, or for times short enough for planar vapor dynamics to be valid the surface pressure $p_{s}$ can be adequately approximated ${ }^{6,7,8}$ with analytic models. Even when a steady state pressure regime is achieved, the observed coupling may show a time dependence. Calculations of the instantaneous mechanical coupling, $p_{S}(t) / I(t)$ and the integrated coupling coefficient $C(t)$, defined as

$$
c(t) \equiv \int_{0}^{t} p\left(t^{\prime}\right) d t^{\prime} / \int_{0}^{t} I\left(t^{\prime}\right) d t^{\prime}
$$

are shown in Fig. 2 as a function of time. Vaporization begins at time $\tau_{v}$. These calculations are for carbon phenolic targets irradiated by $1 \mathrm{MW} / \mathrm{cm}^{2}$ of $10.6 \mu \mathrm{m}$ radiation. ${ }^{9}$ It takes ten times as long to approach the steady state pressure as it does to initiate vaporization; and the integrated coupling coefficient increases even more slowly.

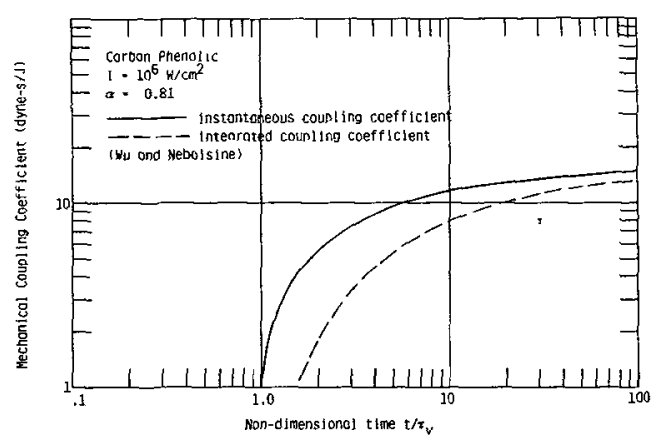

Fig. 2 Mechanical coupling coefficient for carbon phenolic irradiated by $\mathbf{1 0 . 6} \mu \mathrm{m}$ radiation, from Ref. 9. 
Just as ignition of a vapor plasma modifies thermal coupling to a surface, it also alters the mechanical coupling. In an air environment, the laser surface interaction proceeds via LSC waves or laser-supported detonation (ISD) waves ${ }^{10}$; they are discussed under pulsed coupling interactions. In a vacuum, however, the plasma is confined entireIy to the vapor. The mechanical coupling of lasers to surfaces through vapor plasma can be modelled by relating the thickness of the plasma to the absorption depth of the laser radiation in the vapor. 11 The steady state coupling coefficient for a vacuum plasma decreases with intensity, whereas the steady state coupling from vaporization increases with intensity.

\section{PULSED INTERACTION PHENOMENOLOGY}

The phenomenology of the interaction of a pulsed $10.6 \mu \mathrm{m}$ pulse with surfaces depends not only on the average intensity of the pulse, but also on the temporal variations. A typical temporal pulse shape is sketched in Fig. 3. At the leading edge of the pulse there is a gain switched spike followed by a lower intensity tail. The spike lasts only 100-400 ns but its peak intensity is usually two to eight times larger than the average intensity of the tail. The tail, which carries most of the energy, typically has a duration of 3-40 $\mu \mathrm{s}$.

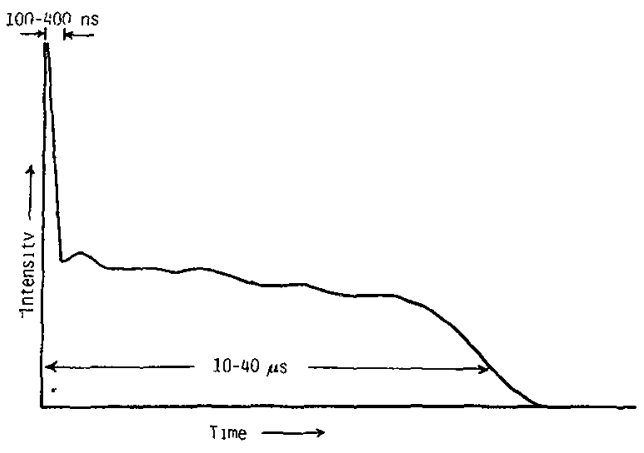

Fig. 3 sketch of 10.6 um temporal pulse shape.
Air plasmas can be created above surfaces by the spike. This process is called prompt ignition to distinguish it from the breakdown of the products of bulk vaporization which was discussed earlier. Bulk vapor breakdown cannot occur until enough time has elapsed for the surface to reach the vaporization temperature. The spike contains insufficient energy to cause bulk vaporization, instead, ignition takes place rapialy from localized defects which vaporize and break down. ${ }^{12,13}$ The spike, then, controls whether or not an air plasma is formed. The threshold for prompt ignition from aluminum is estimated to be a spike fluence ${ }^{12}$ of $1.7 \mathrm{~J} / \mathrm{cm}^{2}$, and a spike intensity ${ }^{14}$ of $10-30 \mathrm{MW} / \mathrm{cm}^{2}$. Typical high energy pulses meet these requirements when the average intensity in the tail is approximately $1 \mathrm{MW} / \mathrm{cm}^{2}$. If no plasma is ignited during the spike, the tail of the pulse is absorbed directly by the surface, and the thermal coupling coefficient is given by the intrinsic absorptivity. If a plasma is initiated, the subsequent interaction proceeds via LSC wave for low average intensity (less than $8 \mathrm{MW} /$ $\left.\mathrm{cm}^{2}\right)^{15,16}$ and an LSD wave at high intensity. As a consequence of the ignition process and the presence of the target surface, a precursor shock precedes the ISC wave except at the lowest intensities. When the LSC wave has propagated far enough for twodimensional effects to dominate the plasma flow in the vicinity of the surface, the plasma configuration resembles that shown schematically in Fig. 4a. The LSC wave propagating into the air behind the precursor shock induces a flow toward the target. Close to the surface the flow resembles a stagnation point flow. A stagnation point boundary layer analysis must be matched to a correct model for the LSC wave propagating away from the surface in order to obtain the temperature and pressure distribution in the plasma, the radiative transport to the tar- 
get and the conductive energy transfer.

At high laser intensities, greater than $8 \mathrm{MW} /$ $\mathrm{cm}^{2}$ for $10.6 \mu \mathrm{m}$ Iaser radiation, a laser-supported detonation (LSD) wave is ignited. A simplified model of the plasma configuration resulting from LSD wave ignition is shown in Fig. 4b. The laser beam absorption takes place in a thin zone of hot, high pressur air behind the detonation wave. since the detonation wave drags air away from the surface, expansion fans form to satisfy the boundary conditions of zero particle velocity at the target surface. One-dimensional gas dynamics can be matched to detonation and planar blast wave theory to describe this aspect of the flow field, and cylindrical blast-wave theory can be utilized to partically account for two-dimensional effects. ${ }^{16}$ An unsteady

a)
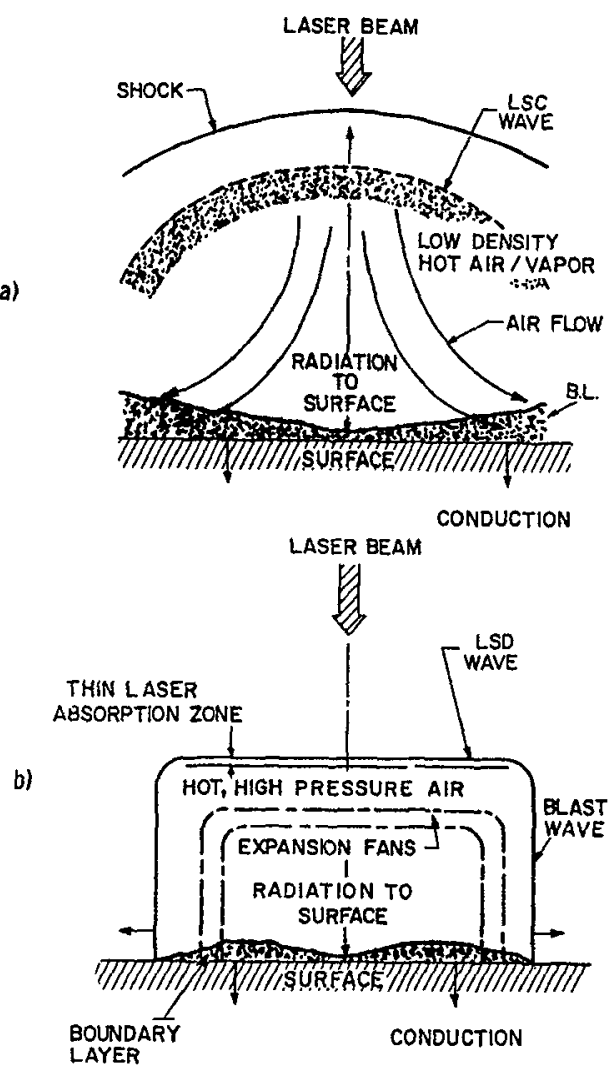

Fig. 4 Sketch of laser absorption wave plasma dynamics (a) LSC wave, (b) LSD wave. From Ref. 19. boundary layer forms on the surface; it resembles the boundary layer behind a propagating shock wave as the cylindrical blast wave spreads out over the target. Energy is transferred from the plasma to the target through this boundary layer by radiation and conduction.

5. ISD WAVE COUPLING: RADIAL EXPANSION EFFECTS In one-dimension the thermodynamic properties behind a true LSD wave is predicted by the Raizer theory. ${ }^{10}$ The analysis of Pirri ${ }^{16}$ gives the conditions above the surface and the time history of the plasma properties as the LSD wave propagates away from the surface. At $10.6 \mu \mathrm{m}$ for an intensity of $20 \mathrm{MW} / \mathrm{cm}^{2}$, the temperature and pressure above the surface are predicted to be $9000^{\circ} \mathrm{K}$ and $53 \mathrm{~atm}$. , respectively. Under these conditions the ratio of the energy transferred from the plasma to the target by radiation and conduction to the laser energy will be less than $1 \%$ for laser pulse times of the order of tens of microseconds. ${ }^{14}$

In order to calculate the total coupling coefficient, plasma spreading must be included. The plasma remains approximately one-dimensional until the expansion fans from the edge of the spot reach to axis of symetry. This time is approximated by the beam radius divided by the speed of sound in the plasma. The pressure decay can be approximated by cylindrical blast wave theory ${ }^{16}$ for time scales greater than two-dimensional time scale. In the vicinity of the surface there is no laser absorption; the plasma properties are determined from isentropic expansion relations. The energy transfer at any instant of time is the sum of the boundary layer heat transfer and radiation contributions.

Calculations $^{14}$ for a two-dimensional LSD wave plasma with a laser intensity of $15 \mathrm{MW} / \mathrm{cm}^{2}$, a spot radius of $1 \mathrm{~cm}$ and a pulse time of 1 ps indicate 
that: (1) radiative energy transfer is minimal, (2) boundary layer energy transfer dominates, as the plasma spreads out over the target, for times up to $1000 \mu \mathrm{sec}$, (3) for the $1 \mu \mathrm{sec}$ pulse the total coupling coefficient is approximately 25\% but is a result of energy spread out over a large area compared to the spot area, and (4) as the laser intensity is increased, the total coupling coefficient tends to remain constant, but the energy is spread out over a larger agea. Therefore, for short laser pulses the total coupling coefficient is significant; however, the local energy transferred into the target is not greater than would be obtained if no plasma was formed. Finally, for this calculation the coupling coefficient varied inversely with pulse time.

The effect of plasma spreading on energy transfer in the LSD regime has been experimentally observed, 17,18 and the thermal coupling is independent of intensity for large targets. 17 Total thermal coupling of a pulsed $10.6 \mu \mathrm{m}$ laser to nickel as measured by Hall et al., 18 is shown in Fig. 5. The nominal laser pulse time is $6 \mathrm{\mu sec}$. The total coupling increases aramatically after a plasma is ignited, but the coupling decreases at higher incident fluence because the plasma expands beyond the target.

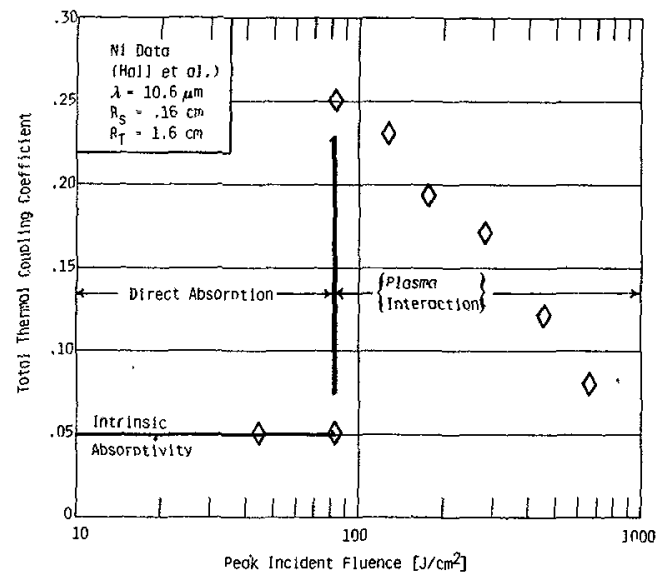

Eig. 5 Thermal coupling of $10.6 \mu \mathrm{m}$ radiation to nicke1. Data from Ref. 18.
6. ISC WAVE REGIME: ENHANCED LOCAT COUPLING Ignition of an LSC wave can lead to enhanced local thermal coupling for aluminum surfaces. ${ }^{1}$ It has been shown that enhanced thermal coupling to aluminum is a result of energy transferred by radiation from the hot, high pressure, laser-supported plasma adjacent to the target. $5,19,20,21$ A sketch of the one-dimensional ISC wave plasma configuration is shown in Fig. 6. The laser is incident from the right hand side. The gain switched spike ignites an air plasma next to the target surface, and the expansion of the plasma drives a precursor shock into the air. The laser absorption zone propagates into the shocked air; the absorption occurs essentially at constant pressure and the propagation of the absorption zone is controlled by conduction and radiative transport from the hot plasma.

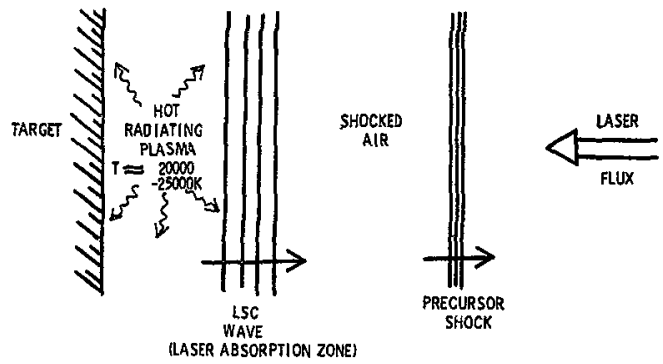

Fig. 6 One-dimensional LSC wave configuration.

The LSC wave propagates into the shocked air at slow speed, and a large fraction of the energy is used to heat the plasma to a temperature of approximately $20000^{\circ} \mathrm{K}$. This hot plasma is capable of radiating in spectral regions less than $1250 \AA$ which are well absorbed by the aluminum targets. 20,21 The expansion of the air as it is heated acts as a piston which maintains the precursor shock.

Efficient local thermal coupling to the surface requires three criteria to be met: (1) a 
plasma must be ignited adjacent to the surface: the plasma must be an LSC wave, and (3) the onedimensional configuration illustrated in Fig. 6 must be maintained throughout the pulse. These requirements are sufficient to identify the range of laser parameters corresponding to the enhanced coupling region. The enhanced coupling region is illustrated in Fig. 7. The coordinates of the plot are laser intensity, $I$, and $\hat{\tau}$, which is the laser pulse time $\tau_{p}$ normalized by the time, $\tau_{2 D}$ ' at which radial expansion of the high pressure plasma affects the center of the laser spot. $\left(\tau_{2 D}\right.$ is defined as $\mathrm{R} / \mathrm{a}_{\mathrm{p}}$ where $R$ is the radius of the laser spot and $a_{p}$ is

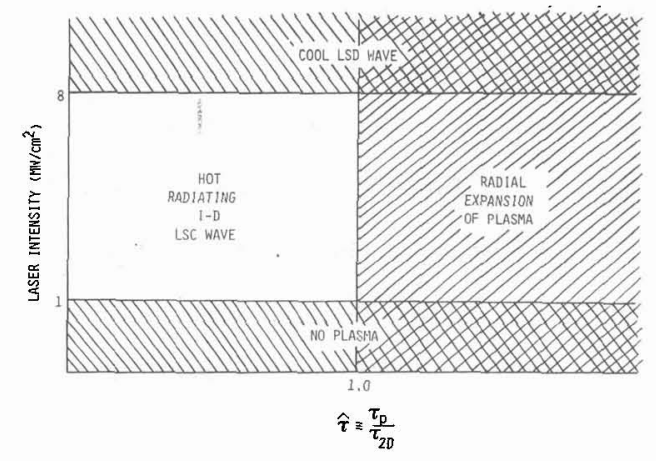

Fig. 7 Region of enhanced local thermal coupling.

the sound speed in the plasma, approximately $4.5 \mathrm{x}$ $10^{5} \mathrm{~cm} / \mathrm{s}$.) Once radial expansion begins, the $d y-$ namics of the plasma no longer maintains the onedimensional character illustrated in Fig. 6; the pressure drops and so does the plasma temperature. As a result, the effectiveness of the plasma in transporting energy via räuiation is rapidly diminished. Thus, the region of $\hat{\tau}>1$ is eliminated from the enhanced coupling region because radial expansion curtails the radiative transport before the laser pulse is terminated. The high intensity region is eliminated because, above the LSD wave transition threșhold of $8 \mathrm{MW} / \mathrm{cm}^{2}$, an ISD wave is produced which has poor coupling. The low inten- sity region, below the plasma ignition threshold of $1 \mathrm{MW} / \mathrm{cm}^{2}$, is eliminated because the gain switch spike corresponding to this laser intensity is not strong enough to create a plasma over aluminum. The remaining area is the enhanced coupling regime.

The 1-D plasma configuration shown in Fig. 6 has been studied theoretically by many investigators and a summary of their contributions is presented in Refs. 14 and 15. To predict thermal coupling the contribution of the radially expanding plasmá shown in Fig. 4a must be included. To make quantitative predictions, a model was synthesized 19,22 in which the early time plasma cynamics was described by the one-dimensional configuration of $\mathrm{Fig}$. 6 and the late time plasma dynamics was represented by blast wave decay laws of the appropriate geometry. The boundary between early time and late time was determined to be the smaller of $\tau_{p}$ and $\tau_{2 D}$. Predictions made with this model agreed with the data within $30 \%$ over most of the range of interest. A complementary approach which was an axially symmetric numerical simulation has also been develop$e d,{ }^{15}$ and there is good agreement between the predictions of the two models.

A comparison of the analytical model predictions and experimental data of Rudder and Augustoni, ${ }^{24}$ and Mckay et al.,,$^{1}$ are shown in Fig. 8 .

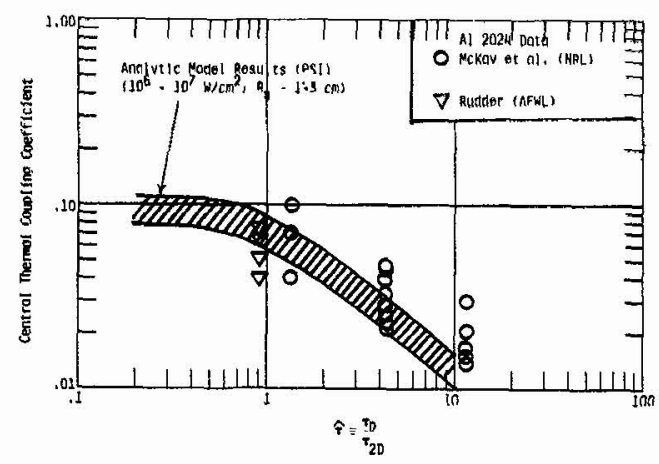

Fig. 8 Central thermal coupling to aluminum by $10.6 \mathrm{\mu m}$ laser radiation. Comparison of data and theory. 
Since the experiments involve a range of intensities, the theoretical curves were calculated for two limiting intensities. The data is generally in agreement with the theory; in particular the predicted decrease in the local thermal coupling with increasing $\hat{\imath}$ is observed. A more detailed comparison of thermal coupling data and theory is discussed later for oblique angles of incidence.

The amount of energy transferred to the surface depends not only on the radiative properties of the plasma, but also on the spectral absorption characteristics of the target. The coupling of metal surfaces other than aluminum is determined by using the appropriate spectral absorptivity.

ISC wave plasmas tend to radiate strongly in the spectral region $\lambda<1250 \AA$. For short pulse times and small spots, and at low intensity most of the radiation is emitted at wavelengths less than $1250 \stackrel{\circ}{\mathrm{A}}$, which all the metals absorb well. There is little difference between the fluence absorbed by various metals for these laser parameters. At longer pulse times, or higher intensity, the radiation in the short wavelength regime becomes saturated and it is controlled by the plasma temperature close to the target, whereas the radiation in the band $\lambda>1250 \mathrm{~A}$, which is transparent, is continually increasing. Even with aluminum, which absorbs long wavelenghs poorly, the Iong wavelength band makes an tmportant contribution for high intensity, long pulse times and large spots. In this regime the coupling to metals other than aluminum, which absorb the long wavelength band better, is enhanced relative to the coupling to Al 2024.

Theoretical predictions of the fluence absorbed by various alloys ${ }^{22}$ indicate that the metals can be arranged into a hierarchy based upon absorbed fluence; the hierarchy can be understood in terms of the spectral absorptivity. Thus, for example, copper and silver, which are better reflectors of $10.6 \mathrm{\mu m}$ radiation than aluminum, are predicted to absorb plasma radiation more strongly than aluminum. For alloys, the strongest absorber is titanium, followed by steel and aluminum.

7. PULSED LASER MECHANICAL COUPLING

Ignition of LSC and LSD waves creates high pressure plasma over the target surface. The resulting impulse delivered to the surface receives a contribution from both the early time planar waves (illustrated in Fig. 6) and the late time two-dimensional waves, shown in Fig. 4. The impulse delivered by an LSD wave was first modelled by Pirri, 6 using analytic methods. More extensive modelling, including numerical simualtions, was performed by Ferriter et al. 24 The impulse from LSC waves has also been calculated ${ }^{25,26}$ based upon the pressure scaling laws used in the thermal coupling calculations. 19

A convenient method of presenting the results is to give the ratio of the predicted impulse over a given area divided by the impulse determined from the planar surface pressure, $p_{S}$, acting on the laser beam area $\pi R_{s}^{2}$ for the pulse time $\tau_{p}$. The results of Bouche et al. ${ }^{26}$ for the ISC wave models are summarized in Fig. 9 as a function of $\hat{\tau}$. Calculations are made for one-dimensional plasma pressures for 10 and $30 \mathrm{~atm}$, which are the limits typically observed experimentally for ISC waves, and the area between them is shaded for all but the infinite plane calculations. The four calculations are: (1) central coupling which uses the pressure predicted at the center of the spot, (2) coupling over the laser spot which includes the spatial variation in pressure over the spot, (3) coupling over the laser spot plus shock annulus which includes the contribution from the area covered by the expand- 
ing radial shock before the end of the pulse, and

(4) coupling to a infinite plane which includes the total impulse delivered by the expanding plasma after the pulse terminates.

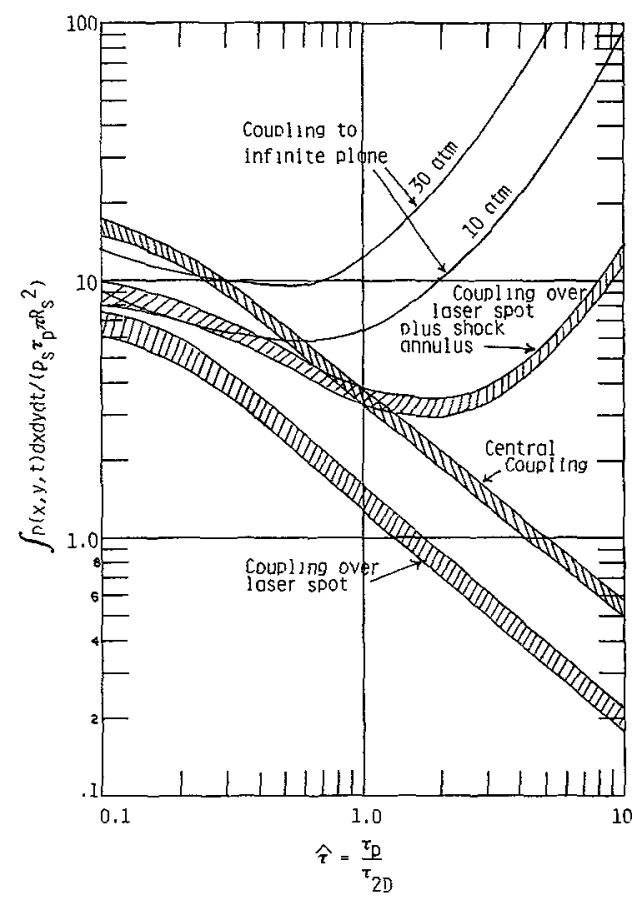

Fig. 9 Predictions of mechanical coupling by LSC wave plasmas. From Ref. 26.

The local coupling to the spot or the center of the spot is enhanced at low $\hat{\tau}$ but is diminished at large $\hat{\tau}$; however, the total coupling is always enhanced.

\section{EXTENSION ANGLE OF INCIDENT AND} AMBIENT PRESSURE

The interaction of pulsed $10.6 \mu \mathrm{m}$ lasex pulses with aluminum surfaces, which are at on oblique angle to the laser beam, has been studied both theoretically 22,27 and experimentally. 28 The goemetry of the interaction at oblique angles of incidence is sketched in Fig. 10. The early time configuration, as viewed from a plane defined by the incident laser beam direction and the target normal, is shown in Fig. 10A. The plasma at the center of the target has no knowledge of the edges at early time; therefore, the plasma dynamics is one-dimensional perpendicular to the target. The plasma configuration is identical to Fig. 6- a precursor shock followed by a laser absorption zone - except that the laser beam is incident at a different angle. The early time behavior can still be modelled as a planar ISC wave if the laser intensity $I$ is changed to the projected intensity I cos $\theta$, and the laser absorption coefficient $k_{L^{\prime}}$ is re-

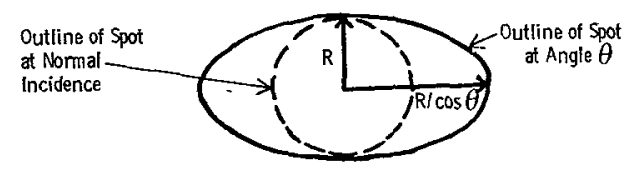

Fig. 10 Angle of incidence geometry. (a) Crosssectional view, (b) target plane view.

placed by the coefficient $k_{L} / \cos \theta$ appropriate for describing laser beam attenuation perpendicular to the target.

The onset of lateral expansion at the center of the spot is determined by a competition between the time for a rarefaction wave generated at the edge of the target spot to reach the center and the time for the laser-supported absorption wave at the center of the target to propagate vertically (in Fig. 10a) to the edge of the laser beam. A view of the laser beam in target plane is shown in Fig. 10b. The characteristic time for lateral expansion along the semi-minor axis is $R / a_{p}=\tau_{2 D^{\prime}}$, where $a_{p}$ is the speed of sound in the plasma; this is the same time scale that controls the radial 
expansion for normal incidence. Only at a later time, characterized by $\tau_{3 D}$, does motion along the semi-major axis begin. The blast wave decay laws for time $t$ are chosen to represent two-dimensional motion for $\tau_{3 D}>t>\tau_{2 D}$ and three-dimensional motion for $t>\tau_{3 D}$. The expansion is represented by powered or unpowered blast wave decay laws according to whether or not the laser is still on.

A comparison between experimental data, ${ }^{28}$ and theoretical predictions 22,27 for the fluence absorbed by Al 2024 targets are shown in Fig. I1, for a nominal laser beam intensity of $1.5 \mathrm{MW} / \mathrm{cm}^{2}$. The beam area is $40 \mathrm{~cm}^{2}$ and the pulse time is $10 \mu \mathrm{s}$. The agreement between data and theory is quite good except for a few data points at normal incidence which are marked by lines to indicate that there was poor plasma ignition. This good agreement supports the original model as well as the extension to angle of incidence.

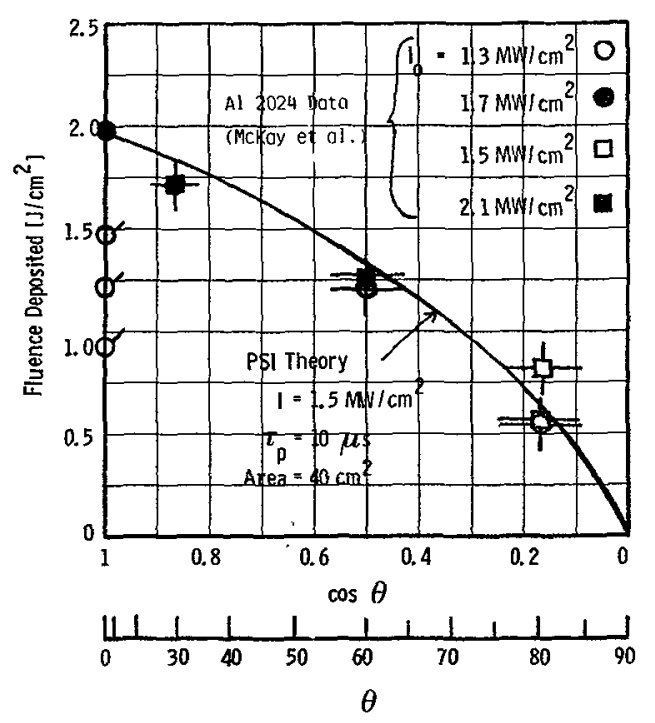

Angle of Incidence

Fig. 11 Fluence deposited in Al 2024 by $10.6 \mu \mathrm{m}$ radiation incident at an angle.

Both the data and the theory show large coupling at large angles of incidence $\left(\theta>74^{\circ}\right)$. The absorbed fluence is twice the value predicted by naively scaling the fluence absorbed for $\theta=0^{\circ}$ by the factor $\cos \theta$. The LSC wave thermal coupling coefficient increases as the projected intensity is reduced. The data also shows that plasma ignition threshold is controlled by the beam intensity, not the projected intensity. 28

The theoretical prediction for the surface pressure generated by the LSC wave is plotted as a function of angle of incidence in Fig. 12. The experimental data ${ }^{28}$ agree quite well with the theoretical predictions lexcept for the data point which is flagged because of poor ignition). The theoretical calculations of impulse imparted to a surface

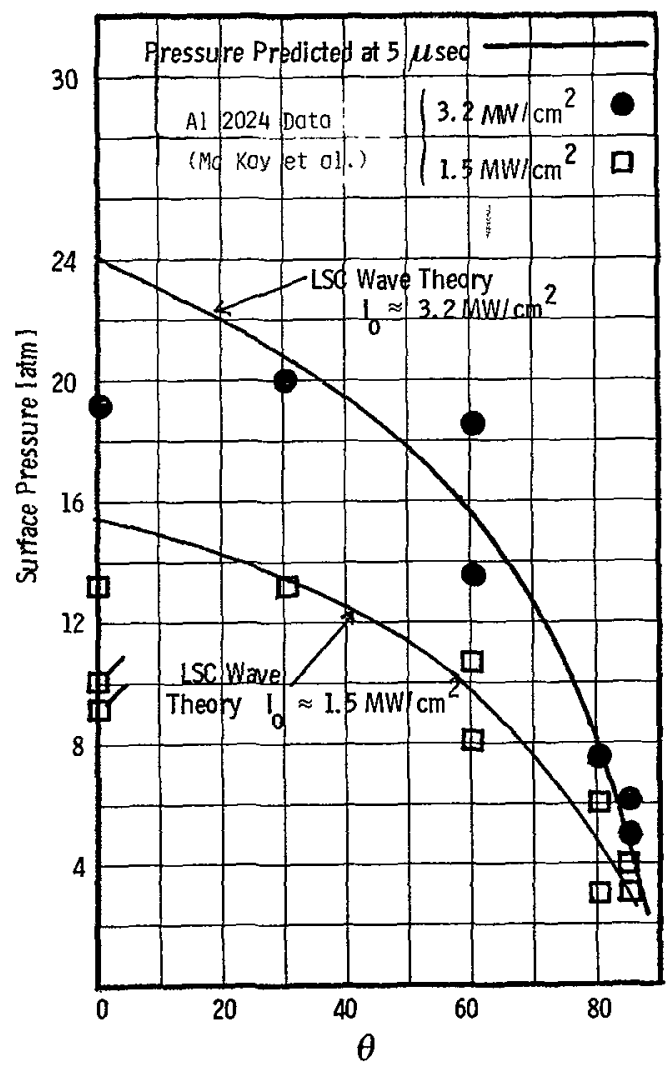

Angle of Incidence

Fig, 12 Surface pressure for $10.6 \mathrm{~mm}$ radiation incident at an angle.

predict that there is little degradation in predicted impulse between $\theta=0^{\circ}$ and $\theta=60^{\circ}$; the arop in surface pressure is partially compensated by 
the slower pressure decay as $\theta$ is increased. At $\theta=75^{\circ}$, the projected fluence is only $1 / 4$ of the normal fluence, but the delivered impulse for $\hat{\mathrm{t}}=1$ is $70 \%$ of the value for normal incidence.

The plasma impulse coupling is predicted to vary as the ambient pressure to the $1 / 3$ power. $10,14,15$ The decrease has been observed experimentally in the LSD wave regime, although the peak pressures measured fall below 30 percent below the predicted values. 29 Experimental data indicate that the thermal coupling by radiative transport from LSC wave plasma remains approximately constant as the pressure is reduced, as Iong as a well-developed plasma is formed. However, as the ambient pressure drops a threshold is reached below which an air plasma cannot be created. The thermal coupling at pressures below the air threshold is accomplished by direct absorption; at least for low laser intensities. However, it has been observed by McKay and Schriempf ${ }^{31}$ that at high intensity it is possible to ignite a vacuum plasma which enhances the thermal coupling. This plasma is not the result of bulk vaporization, ${ }^{31}$ instead it apparently is created by defect vaporization and is, therefore, different than the $\mathrm{CW}$ vapor plasma mentioned in Section 3.

\section{PULSED $3.8 \mu \mathrm{m}$ COUPLING CONSIDERATIONS}

Although both $10.6 \mu \mathrm{m}$ and $3.8 \mu \mathrm{m}$ pulsed laser radiation exhibit enhanced thermal coupling to highly reflective metal targets, the mechanisms by which the coupling is achieved may be different.

The DF laser pulse, from the Boeing photolytically initiated laser, ${ }^{32}$ which is shown in Fig. 13, does not have a leading edge spike and it has a relatively long rise time to approximately .85 usec. Plasma ignition occurs in the midale of the pulse. $2,32,33$ As a result, the physics of the interaction between the laser and the target cannot be categorized for the duration of the whole pulse, as either direct laser absorption or plasma radiative transfer.

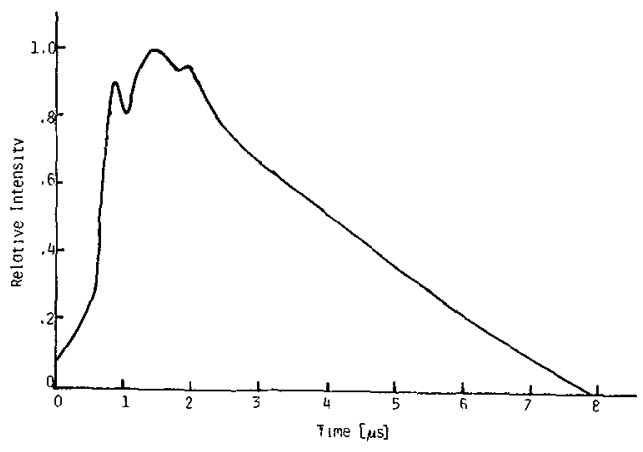

Fig. 13 Sketch of DF laser pulse shape. ${ }^{32}$

Nor can direct.absorption of DF laser radiation be simply characterized by the intrinsic room temperature absorptivity as it can be for $10.6 \mu \mathrm{m}$ pulsed radiation. For $3.8 \mu \mathrm{m}$ radiation the initial absorptivity of Al 2024 is larger, namely .05, and the peak intensity is usually greater than $10 \mathrm{MW} /$ $\mathrm{cm}^{2}$. The absorbed heat flux in the direct absorption regime is more than an order of magnitude larger than at $10.6 \mathrm{\mu m}$. This rapid heat transfer can raise the target surface temperature significantly during the pulse. To understand the coupling when no plasmas are formed, the effects of target heating and mass loss must be considered just as they are in CW interactions.

It is not known a priori whether plasma ignition by pulsed $3.8 \mu \mathrm{m}$ radiation is associated with localized defects, as it is for $10.6 \mu \mathrm{m}$ radiation, or whether it results from breakdown of the vapor produced by bulk evaporation of the surface as it does in $\mathrm{CW}$ interactions. Since inverse Bremsstrahlung absorption by electrons scales as wavelength squared, the threshold intensity for defect initiation of plasma is higher for DF laser pulses. However, the larger bulk heating rate experienced by 
the metal under $3.8 \mathrm{\mu m}$ irradiation may lead to bulk vaporization of the target during the pulse; then plasma initiation can proceed by breakdown of the bulk vapor as it does in $\mathrm{CW}$ interactions.

The threshold for LSD waves for short pulses of $3.8 \mu \mathrm{m}$ radiation is estimated to be $40-50 \mathrm{MW} /$ $\mathrm{cm}^{2} \cdot 14,15$ The LSC wave plasmas at $3.8 \mathrm{\mu m}$ are supported by much higher intensities than at $10.6 \mu \mathrm{m}$; in consequence the plasmas are hotter, at higher pressure, and the radiative transfer to the surface is a factor of ten larger.

A layer of metal vapor may be created hetween the plasma and the target either as a result of the ignition process or as a consequence of the high heat flux. The vapor absorbs the plasma radiation, thus it interferes with energy transport to the surface until it is raised to high temperatures where it also will radiate. However, metal vapors tend to radiate preferentially in the longer wavelength bands, which reduces the thermal coupling to aluminum.

Recent data ${ }^{32}$ on the fluence deposited by pulsed $3.8 \mu \mathrm{m}$ laser radiation interacting with aluminum surfaces is shown in Fig. 14. The shaded area

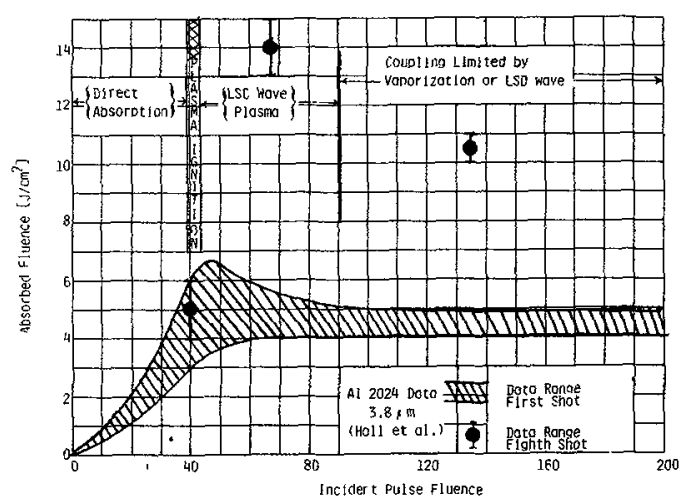

Fig. 14 Fluence deposited in Al 2024 by $3.8 \mathrm{\mu m}$ radiation. Data from Ref. 32 . represents the range of values observed for the first shot on a fresh aluminum surface. The circles with bars represent the data range observed for the eight shot on the surface. The multiple pulse effect was investigated at only three fluences. Plasma ignition occurs between at an average fluence of $40-50 \mathrm{~J} / \mathrm{cm}^{2}$ (the spatial peak in the fluence is about $70 \mathrm{~J} / \mathrm{cm}^{2}$ ). For fluences less than $40 \mathrm{~J} / \mathrm{cm}^{2}$, the interaction proceeds by direct absorption. Above $40-45 \mathrm{~J} / \mathrm{cm}^{2}$, direct absorption occurs at the beginning of the pulse, but in the midale of the pulse a plasma is ignited and the subsequent interaction is mediated by an LSC wave plasma. The data indicates that there is 1ittle enhancement associated with plasma formation for the first shot, but the enhancement is substantial on subsequent shots in the plasma regime. The increase of coupling with the number of shots is not completely understood, but it appears to be related to the increase in the surface absorptivity of the target which occurs as the result of surface damage by prior irradiations. 32

At high fluences, say above $80 \mathrm{~J} / \mathrm{cm}^{2}$, the taxget can vaporize and the vapor layer curtails radiative transport. Creation of local LSD waves could also cause the reduction in absorbed fluence, but the multiple pulse enhancement argues against the interpretation.

Although the basic interactions which govern 3.8 $\mu \mathrm{m}$ pulsed laser interactions with metal surfaces appear to be a combination of those observed in $\mathrm{CW}$ interactions and those found in pulsed 10.6 $\mu \mathrm{m}$ interactions, a detailed understanding of the data shown in Fig. I4 is still lacking.

\section{INTERACTION WITH NON-METALS}

The types of interactions which have been observed in metals can also occur for non-metals such as ceramics and fiberglass. The major difference 
is that the non-metals can absorb radiation indepth. Simple theoretical models have been used to analyze data from single pulse experiments in fresh surfaces of fiberglass (Cordopreg ${ }^{\circledR}$ E-glass), slip cast fused silica (SCFS) and pyroceram.

Figure 15 shows the pulse data for the maximum value of the surface pressure observed during the interaction of pulsed $10.6 \mathrm{\mu m}$ radiation with non-metals. 33 Also shown are the theoretical predictions for the pressure, based upon the synthesis of models described below. The theoretical predictions are in good agreement with the data, thus lending credence to the phenomenology underlying the predictions.

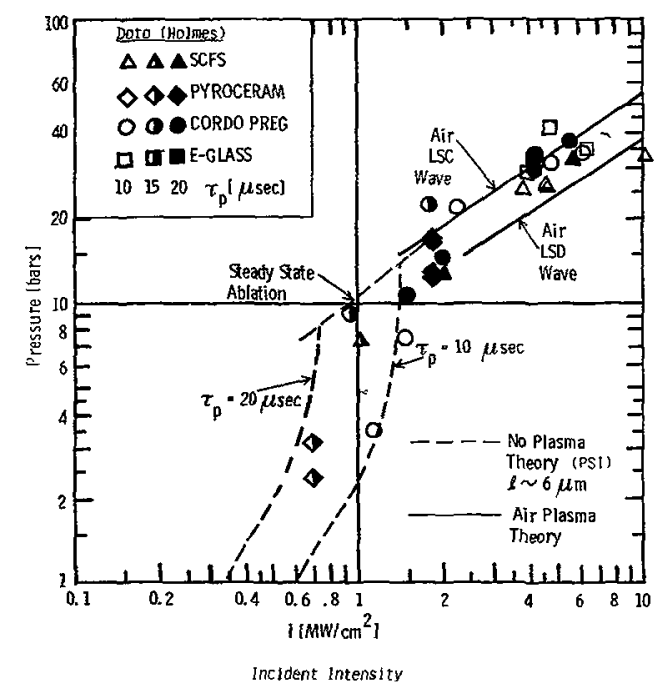

Fig. 15 surfaces pressure for $10.6 \mathrm{Hm}$ radiation on non-metals. Data from Ref. 33.

At laser intensities above $2 \mathrm{MW} / \mathrm{cm}^{2}$, the surface interaction is dominated by the prompt formation of an air plasma above the surface, and the surface pressure and radiative transfer to the surface can be determined from the LSC wave model described in section $6 .^{23}$

Theoretical calculations predict that vaporization induced by plasma reradiation has only only a negligible effect upon the surface pressure gen- erated by ISC waves over aluminum targets; that is, the pressure is given by the LSC wave predictions below $4 \mathrm{MW} / \mathrm{cm}^{2}$, by ISD wave predictions above $8 \mathrm{MW} / \mathrm{cm}^{2}$, and by a transition from ISC wave values to LSD wave values between $4 \mathrm{MW} / \mathrm{cm}^{2}$ and $8 \mathrm{MW} / \mathrm{cm}^{2}$. The pressure data for intensities above $2 \mathrm{MW} / \mathrm{cm}^{2}$ are shown in Fig. 15 and they are consistent with the theoretical predictions.

At laser intensities below $2 \mathrm{MW} / \mathrm{cm}^{2}$, the surface interaction of the virgin target is dominated by direct absorption of the laser by the target with an in-depth absorption length of $6 \mathrm{\mu m}$. Pressure is generated as the result of vaporization of the glass fibers. The time resolved surface pressure traces indicate that the pressure builds up slowly, rather than promptly as would be expected if a plasma were ignited. ${ }^{33}$ The maximum surface pressure data, shown in Fig. 15, show an abrupt fall-off as a function of Eluence. This behavior is inconsistent with vaporization induced by surface absorption of the laser, but it is in good agreement with vaporization models based on in-depth absorption with an absorption length of $6 \mathrm{\mu m}$. As the laser pulse fluence is increased beyond the threshold values for pressure generation, the maximum surface pressure is consistent with the predictions based on steady state target, vaporization.

At intensities below plasma threshold, the energy remaining in the target after the pulse, called the residual energy, is limited by the onset of rapid vaporization. Theoretical analysis, 34 predict a maximum residual energy of $6 \mathrm{~J} / \mathrm{cm}^{2}$ for a $6 \mu \mathrm{m}$ absorption depth and target initially at room temperature.

The response of fiberglass to irradiation 22,35 by pulsed lasers indicates that the useful energy for bulk material heating on a multiple pulse basis 
is limited to less than $7 \mathrm{~J} / \mathrm{cm}^{2}$. However, the same multiple pulse experiments indicate that the plasma threshold for a previously irradiated target may decrease to below $1 \mathrm{MW} / \mathrm{cm}^{2}$.

\section{SUMMARY}

The thermal and mechanical coupling of laser beams to materials varies strongly, as the interaction phenomenology changes. For direct absorption, the instantaneous thermal coupling is given by the target surface absorptivity, but the total thermal coupling includes effects from target heating and mass loss. Whenever plasmas are created, either by bulk vapor breakdown or by defect induced breakdown, the laser energy is absorbed in the plasma, and mechanical and thermal coupling is determined by the plasma properties. For LSD wave plasmas, thermal coupling is dominated by plasma radial expansion and enhanced total thermal coupling can occur; for LSC wave plasmas, radiative transport dominates the transport and enhanced local coupling is observed. Mechanical coupling results from vaporization in the direct absorption regime, and from the high pressure plasma in the plasma mediated coupling regime. The plasma phenomena which are observed for $\mathrm{CW}$ interactions and pulsed 10.6 $\mu m$ interactions, also occur for oblique angles of incidence, for pulsed $3.8 \mathrm{um}$ laser radiation, and for the interaction with non-metal materials, however, the laser parameters which delineate the interaction regimes, as well as the magnitude of the effects, are different.

\section{RESUME}

Le couplage thermique et mécanique d'un faisceau laser avec des matériaux varie en fonction de l'intéraction qui a lieu. si l'absorption est directe, la couplage thermique instantané est déterminé par l'absorptivité de la surface mais le couplage intégré comprend les effets de chauffage de la cible et de perte de masse. Quand il y a création d'un plasma, par claquage soit dans le gros de la vapeur soit initié par des défauts de surface, l'énergie est absorbée par le plasma et les couplages thermique et mécanique sont determinés par les propriétés de la vapeur. Quand il y a formation d'un onde de détonation (ISD), le couplage thermique est dominé par I'expansion radiale du plasma: il peut $y$ avoir augmentation du couplage thernique global. Quand il $y$ a formation d'une onde de combustion (ISC), le transport thermique est principalement par radiation: on observe une augmentation du couplage local. Le couplage mécanique provient de la vaporisation de la surface dans le régime à absorption directe et de la pression éleveé du plasma dans le régime à couplage indirecte. Les mêmes phénomènes que I'on abserve dans les intéractions avec lasers continus et lasers $\mathrm{CO}_{2}$ à impulsion on lieu aussi dans le cas d'incidence oblique, dans le cas d'un laser impulsionnel à $3.8 \mu \mathrm{m}$ ou dans le cas de matériaux non métalliques: les paramètres du laser qui limitent les différents régimes d'intéraction et l'orare de grandeur des effets sont cependant différents.

REFERENCES

1. McKay, J. A., Stegman, R. L., and Schriempf, J. T., "Thermal Effects of Pulsed Laser Ixradiation," 2nd DoD High Energy Laser Conference Proceedings, Col. Springs, Col., Nov. 1976.

2. Nichols, D, B. and HaIl, R. B.: "mhermal Coupling of 2.8-Micron Laser Radiation to Metal Tarujets." Paper No. VI-6 presented at AIAA Conference on Fluid Dynamics of High Power Lasers, October 30-November 2, 1978, Carabridge, MA.

3. Klosterman, Elliot Lee, "Expcrimentał Investigation of Subsonic Laser Absorption Wave Initiation from Metal Targets at 5 and 10.6 Hm," Mathematical Sciences Northwest, Inc., MSNW Report No. 75-123-2, March, 1975.

4. Thomas, P. D. and Musal, H. M., "A Theoretical Study of Laser-Target Interaction," Final 
Technical Report No. I.MSC-D352890, Lockheed Missiles and space Company, Inc., Palo Alto, CA, August 1973.

5. Raizer, Yu P., Soviet Physics JETP, 31, 1148 (1970).

6. Pirri, A. N., "Analytic Solutions for LaserSupported Combustion Wave Ignition Above Surfaces," AIAA Paper 76-23, Washington, D.C., Jan. 1976 .

7. Thomas, P. D. and Musal, H. M., "A Theoretical Study of Iaser-Target Interaction," Lockheed Palo Alto Research Iaboratory Report LMSCD 352890, Palo Alto, CA, Aug. 1973 and Part II, LMSC-D401354, April 1974.

8. Pirri, A. N., "Analytical Solutions for Initiation of Plasma Absorption Above Laser-Irradiated surfaces," Technical Report No. PSI TR15, Physical Sciences Inc., Woburn, MA, oct. 1974.

9. Wu, P. K. and Nebolsine, P. E., AIAA Journal, 15,751 (1977).

10. Raizer, Yu P., Soviet Physics JETP, 21, 1009, (1965).

11. Basov, N. G. et al., Soviet Physics JETP, 27, 575 (1968)

12. Walters,C. T., Barnes, R. H., Beverly, R. E. III, J. Appl. Phys. 49, 2937 (1978).

13. Weyl, G. Pirri, A., and Root, R., "Laser Ignition of Plasma off Aluminum Surfaces," AIAA Paper 80-1319, Snowmass, CO, July 1980.

14. Pirri, A, N., Kemp, N. H., Root, R. G., and Wu, P. K. S., "Theoretical Laser Effects Studies," Final Report, PSI TR-89, Physical Sciences Inc., Wobuxn, MA, Feb. 1977.

15. Boni, . A., Su, F. Y., Thomas, P. D., and Musal, H. M. , "Theoretical Study of Laser-Target Interactions," Final Tech. Rept., SAI 77-77a567-IJ, Science Application Inc., LaJolla, CA, May 1977.

16. Pirri, A. N., Phys. Fluids 16, 1435 (1973).

17. McKay, J. A. et al, J. Appl. Phys. 50, 3231 (1979).

18. Maher, W. E., and Hall, R. B., J. Appl. Phys., 49,2254 (1978).

19. Pirri, A. N., Root, R. G., and Wu, P. K. S. AIAA Jour. 16, 1296 (1978).

20. Mitchel1, R. W. et al., J.Q.S.R.T. 20, 519 (1978).

21. Root, R. G. and Pirri, A. N., "Theoretical Analysis of Radiation from Laser Produced Plasma," AIAA Paper 79-1489, Williamsburg, VA, July 1979.

22. Root, R. G., Pirri, A. N., Wu, P. K. S., and Gelman, H., "Analysis of Laser Target Interaction," Final Report, PSI TR-170, Physical Sciences Inc., Woburn, MA, March 1979.

23. Rudder, R. R. and Augustoni, A. I., "Thermal
Deposition Experiments with Miscrosecond Duration $\mathrm{CO}_{2}$ Laser Radiation," Laser Digest-Summer 1975, AFWL-TR-75-229, Air Force Weapons Laboratory, Kirtland Air Force Base, NM, Oct. 1975.

24. Ferriter, N. et al., AIAA Jour. 5, 1597 (1977).

25. Reilly, J. P., Ballantyne, A., and woodroffe, J. A., AIAA JournaI, 17, 1098 (1979).

26. Reilly, J. and Bouché, E., W. J. Schafer Assoc., wakefield, MA, (private communiation).

27. Geiman, H., Pirri, A. N., Root, R. G. and Wu, P. K. S., "Coupling of Non-Normally Incident Pulsed Laser Flux to Metals Surfaces in an Air Environment," AIAA Paper 79-0251, New Orleans, LA, January 1979.

28. MCKay, J. A. et a1., App. Phys. Letters, 36, 125 (1980)

29. Beverly, R. E., III, and Walters, C. T., J. App. Phys. 47,3485 (1976).

30. McKay, N, and Research Iab., Washington, D.C., (private communication).

31. Mckay, J. A. and Schriempf, J. T., App. Phys. Lett. 35, 433 (1979).

32. Maher, W. E., and Hall, R. B., Bull. APS 24 1003 (1979).

33. Holmes, B. S., "Pulsed Laser Thermal Mechanical Damage Study, Vol 1 - Surface Pressure from LSA Waves," AFWI-TR-80-31, Air Force Weapons Laboratory, Kirtland Air Force Base, NM (Dec. 1979\}.

34. Wu, P. K. and Root, R. G., AIAA Jour. 18, 857 (1980).

35. Ballantyne, A. et al." "Modelling of the Interactions of $10.6 \mathrm{\mu m}$ Pulsed Laser Radiation with Reinforced Plastic Materials, AIAA Paper 80-1318, Snowmass, CO, July 1980. 